\section{Tuberculosis immunodiagnosis: delving below the surface}

\author{
Ajit Lalvani, ${ }^{1,2}$ David W Connell ${ }^{1,2}$
}

It is one hundred and thirty years since Franz Ziehl and Friedrich Neelsen developed the rapid stain for acid-fast bacilli; ${ }^{12}$ accurate point-of-care diagnosis of active tuberculosis (TB) remains a major unmet clinical need. With the sensitivity of the Ziehl-Neelsen stain in sputum less than $50 \%$ and more than $20 \%$ of TB cases negative on both acid-fast stain and culture for Mycobacterium tuberculosis, there has long been a yawning gap in the diagnostic toolkit for TB. New rapid molecular methods have recently improved detection of $M$ tuberculosis nucleic acids in sputum providing diagnostic sensitivity that is much higher than sputum-smear microscopy but lower than culture. $^{3}$

The longstanding diagnostic gap has stimulated decades of research into immunodiagnosis, mostly serological. Although serological tests for TB are point-of-care, they lack diagnostic accuracy and are devoid of clinical utility. After thorough review of the evidence, and on account of their continued widespread misuse in many high-burden countries, WHO recently tried to bury current commercial serological test kits with a negative endorsement warning against their use. ${ }^{4}$

Research into cellular immunodiagnosis has been more fruitful, delivering a tangible advance in clinical practice in the form of interferon-gamma release-assays (IGRA). ${ }^{5}$ IGRA detect $M$ tuberculosis infection, providing a new standard-of-care for diagnosis of latent TB infection (LTBI), but they cannot distinguish active TB from LTBI. Hence, their potential role in evaluation of patients with suspected active TB is limited to that of a possible rule-out test of TB, yet currently available IGRA lack sufficient diagnostic sensitivity for this indication, as discussed elsewhere in this issue of Thorax. $^{67}$

Are there immune responses that differ sufficiently between active TB and LTBI to enable development of an immunodiagnostic test that is specific for active TB?

\footnotetext{
${ }^{1}$ Tuberculosis Research Unit, National Heart and Lung Institute, Imperial College London, London, UK;

${ }^{2}$ Tuberculosis Service, Imperial College Healthcare NHS Trust, St. Mary's Hospital, London, UK

Correspondence to Professor Ajit Lalvani, Tuberculosis Research Unit, National Heart and Lung Institute, Imperial College London, London W21PG, UK; a.lalvani@imperial.ac.uk
}

Quantifying genome-wide host geneexpression yielded a signature of 393 genes whose differential expression between active and latent TB enabled accurate distinction of active TB from most cases of LTBI. ${ }^{8}$ Translating such a genomic signature into a clinically useful diagnostic test suitable for use in routine diagnostic laboratories is a priority, but will be a long and arduous process.

Could we use existing measures of immune responses more intelligently to tease out responses that predominate in active TB versus LTBI? This requires delving below the 'surface' of the humoral immune response to uncover the different components that contribute to the composite read-out that is measured by conventional serological tests. Such tests do not distinguish between antibodies that are currently being produced in vivo in response to an infection and pre-existing antibodies. Serum antibody levels include the cumulative history of antibody responses induced by previous exposure to antigens. However, during an active infection, responding $\mathrm{B}$ cells migrate through the circulation as plasmablasts secreting antibodies specific for the pathogen. By separating out peripheral blood white cells from blood (and hence from serum) it is possible to measure newly secreted antibodies produced by circulating activated plasmablasts in vitro, independently of pre-existing antibodies (figure 1), as was first shown for measurement of vaccine-induced responses.

This method, known as Antibodies in Lymphocyte Supernatant (ALS), was previously used with BCG vaccine as antigen to detect BCG-specific antibodies in TB patients. It showed promising results as a biomarker for active TB in both adults 910 and children. ${ }^{1112}$ In this issue of Thorax, Ashenafi et al evaluated this assay in parallel with the tuberculin skin test (TST) and an IGRA (Quantiferon TB-Gold-in-tube) in adult TB patients (with and without HIV to coinfection) and asymptomatic controls with and without LTBI in Ethiopia.

By comparing these tests with a definite clinical diagnosis of TB, (which was carefully defined by microbiological, cytological and clinical characteristics), they were able to construct receiver-operating characteristic curves and an optimal cut-off for ALS positivity. Using this, they showed that the ALS assay was sensitive (positive in 91\% of all cases of active TB), and could discriminate active TB from LTBI where the test was only positive in $16 \%$ of cases; its specificity was $94 \%$ in asymptomatic controls without LTBI. As expected, and in contrast with ALS, neither the TST nor the IGRA discriminated active TB from LTBI. Additionally, in a subset of patients, they were able to show that the number of $\operatorname{IgG}+$ plasmablasts in peripheral blood mononuclear cells (PBMCs), as determined by flow cytometry, correlated with the titres of BCG-specific IgG antibodies secreted by the cultured PBMCs in the ALS assay.

In the context of the existing literature with ALS in TB, the main advance provided by this study is that the diagnostic sensitivity of this assay is well maintained in HIV coinfection, including advanced immunosuppression. Given the difficulty of reliably diagnosing $\mathrm{TB}$ in HIV coinfection, advances in this patient population are urgently needed. Intriguingly, there was a significant inverse correlation between CD3 T cell numbers and BCG-specific IgG titres among TB patients. This paradoxical correlation of increasing diagnostic sensitivity with advancing HIV-induced immunosuppression is analogous to the detection of the $M$ tuberculosis cell wall component lipoarabinomannan (LAM), in urine, where mycobacterial dissemination in immunosuppression results in increased diagnostic sensitivity. ${ }^{13}$ An interesting pattern is emerging where certain new diagnostic tests for TB may find clinical application in specific subgroups of patients. By contrast with BCG-specific IgG titres, and as expected and previously documented, $M$ tuberculosis-specific interferon-gamma production measured by Quantiferon TB-Gold-in-tube was significantly reduced in HIV coinfection and decreased with declining CD4 T cell numbers.

So is it time to exhume the cadaver recently buried by WHO report? Much remains to be done before any such disinterment can be considered. The results of the current study are not generalisable. The diagnostic sensitivity reported is for a population of TB patients with sputum-smear negative pulmonary $\mathrm{TB}$, pleural $\mathrm{TB}$ and lymph node TB. While including a substantial overall proportion of clinical presentations of $\mathrm{TB}$, the $91 \%$ diagnostic sensitivity cannot be extrapolated to other types of extrapulmonary $\mathrm{TB}$, which represent a significant diagnostic challenge, especially in HIV coinfection.

Although the specificity of the assay was 94\% in asymptomatic controls without 
Figure 1 Antibodies measured in serum include antibodies produced in response to a current infection, as well as antibodies produced in response to previous exposures; the former make up only a small proportion of all the measurable antibodies in serum. By contrast, during an active infection, the proportion of the circulating B cell pool that comprises plasmablasts specific for that infection and actively secreting antibodies is significantly greater than the memory B cell population which represent previous exposure to antigen.

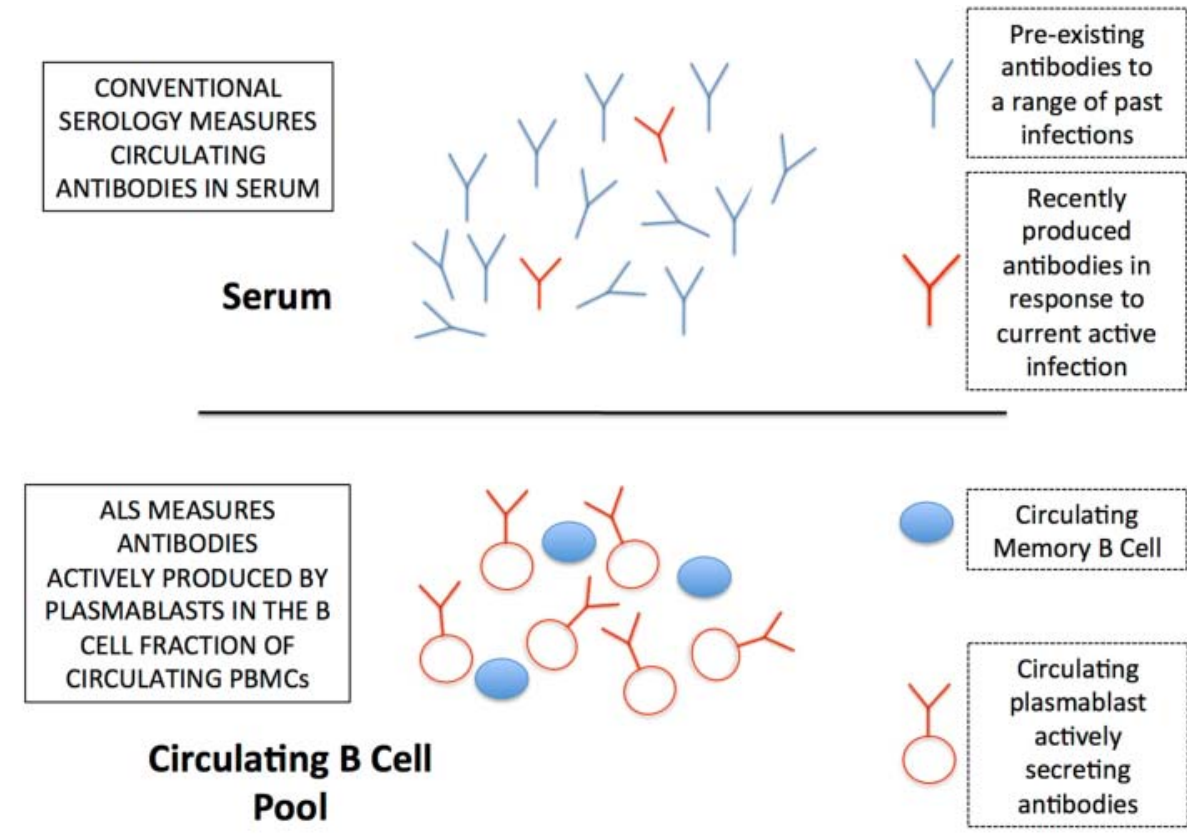

Pre-existing infections

Recently produced antibodies in response to urrent activ infection

actively

antibodies
LTBI, this result is probably an overestimate of the real specificity in clinical practice. The actual target population for this assay is patients with suspected active TB and a high proportion of such patients who turn out not to have active TB will have LTBI. With $16 \%$ of the LTBI group scoring positive in this assay, specificity in a population of real-life TB suspects will, accordingly, be lower than 94\%. Although 12 of the 96 patients with suspected TB in this study turned out not to have TB, the number of false-positive results in this group is not reported. Moreover, non-TB illnesses might also cause false-positive results and these 12 patients were the only group in whom this might have been assessed.

The case-control study design precludes reliable estimates of positive (PPV) and negative predictive values (NPV) as these parameters require the appropriate reallife population in which the test would be deployed, that is, prospectively presenting TB suspects. These values also depend crucially on the prevalence of the target condition (active TB) and confounders that may cause false-positive results (such as LTBI and non-TB illnesses) in the study population.

Hence, the next step rigorously to validate this new immunodiagnostic assay is a large prospective study in routine clinical practice of patients with suspected TB in whom the diagnosis is unknown at the point of recruitment, as has been done for IGRA. ${ }^{14}$ Those patients who transpire to not have TB, but who instead have non-TB illnesses and/or LTBI, will be the appropriate real-life 'control' group, enabling reliable generalisable estimates of diagnostic test performance, PPV and NPV.

If future studies confirm that the diagnostic performance of ALS confers clinical utility in routine practice, there are logistical issues that will need to be addressed. Although the ALS measures antibodies, it is not yet a point-of-care test, requiring $72 \mathrm{~h}$ incubation in sterile tissue culture conditions at $37^{\circ} \mathrm{C}$ with $5 \%$ ambient $\mathrm{CO}_{2}$.

Despite its limitations, this proof-of-principle paper by Ashenafi et al shows what can be achieved by dissecting out the components of the immune response that lie below the surface that is conventionally measured. This is analogous to recent advances in $\mathrm{T}$ cell-based diagnosis, where dissecting out the $\mathrm{T}$ cell subsets that contribute to the interferon-gamma measured by current IGRAs, ${ }^{15}{ }^{16}$ and indeed dissecting out the $\mathrm{T}$ cell subsets contributing to the $M$ tuberculosis-specific $\mathrm{T}$ cell response as a whole, enables differentiation of untreated active TB, treated TB and LTBI. ${ }^{17} 18$

After decades in the doldrums, researchbased development of new TB diagnostics has entered an exciting phase. And using recent advances in immunology to delve beneath the surface of conventional immunological readouts is accelerating to accelerate the development of newer, more informative immunodiagnostic paradigms.

Competing interests $\mathrm{AL}$ is inventor for patents underpinning T cell-based diagnosis. The IFN- $\gamma$ ELISpot IGRA was commercialised by an Oxford University spin-out company (T-SPOT.TB, Oxford Immunotec) in which Oxford University and AL have minority shares of equity and royalty entitlements.

Funding AL is a Wellcome Trust Senior Research Fellow in Clinical Science and a National Institute for Health Research Senior Investigator. DC is a Medical Research Council Clinical Research Training Fellow.

Provenance and peer review Commissioned; internally peer reviewed.

To cite Lalvani A, Connell DW. Thorax 2013;68 204-206.

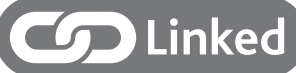

http://dx.doi.org/10.1136/thoraxjnl-2011-201817

Thorax 2013:68:204-206.

doi:10.1136/thoraxjnl-2012-202481

\section{REFERENCES}

1 Ziehl F. Ueber die Farbung des Tuberkelbacillus. Deutsche Med Wochenschr 1883:9:247-9.

2 Neelsen F. Ein Casuistischer Beitrag zur Lehre von der Tuberkulose. Zentrablau fur die medizinischen Wissenschafen 1883:21:497-501.

3 Boehme CC, Nabeta P, Hillemann D, et al. Rapid molecular detection of tuberculosis and rifampin resistance. N Engl J Med 2010;363:1005-15.

4 World Health Organization. Commercial serodiagnostic tests for diagnosis of tuberculosis: policy statement, 2011. http://whqlibdoc. who.int/ publications/2011/9789241502054_eng.pdf (accessed 9 Jan 2013).

5 Lalvani A. Diagnosing tuberculosis infection in the 21st century: new tools to tackle an old enemy. Chest 2007:131:1898-906.

6 Lalvani A, Millington KA. T-cell interferon-gamma release assays: can we do better? Eur Respir J 2008:32:1428-30.

7 Abubakar I, Stagg H R, Whitworth $\mathrm{H}$, et al. How should I interpret an Interferon Gamma Release Assay result for tuberculosis infection. Thorax 2013 [March issue]. 
8 Berry MP, Graham CM, McNab FW, et al. An interferon-inducible neutrophil-driven blood transcriptional signature in human tuberculosis Nature 2010;466:973-7.

9 Raqib R, Rahman J, Kamaluddin AK, et al. Rapid diagnosis of active tuberculosis by detecting antibodies from lymphocyte secretions. I Infect Dis 2003;188:364-70.

10 Rekha RS, Kamal SM, Andersen P, et al. Validation of the ALS assay in adult patients with culture confirmed pulmonary tuberculosis. PLoS One 2011;6: e16425.

11 Raqib R, Mondal D, Karim MA, et al. Detection of antibodies secreted from circulating Mycobacterium tuberculosis-specific plasma cells in the diagnosis of pediatric tuberculosis. Clin Vaccine Immunol 2009;16:521-7.

12 Thomas T, Brighenti S, Andersson J, et al. A new potential biomarker for childhood tuberculosis. Thorax 2011;66:727-9.

13 Dheda K, Davids V, Lenders L, et al. Clinical utility of a commercial LAM-ELISA assay for TB diagnosis in HIV-infected patients using urine and sputum samples. PLoS One 2010;5:e9848.

14 Dosanjh DP, Hinks TS, Innes JA, et al. Improved diagnostic evaluation of suspected tuberculosis. Ann Intern Med 2008;148:325-36.

15 Millington KA, Innes JA, Hackforth S, et al. Dynamic relationship between IFN-gamma and IL-2 profile of Mycobacterium tuberculosis-specific
T cells and antigen load. J Immunol 2007:178: 5217-16.

16 Casey R, Blumenkrantz D, Millington K, et al. Enumeration of functional $\mathrm{T}$-cell subsets by fluorescence-immunospot defines signatures of pathogen burden in tuberculosis. PLoS One 2010; 5:e15619.

17 Harari A, Rozot V, Enders FB, et al. Dominant TNF- $\alpha+$ Mycobacterium tuberculosis-specific CD4+ $T$ cell responses discriminate between latent infection and active disease. Nat Med 2011;17:372-6.

18 Day CL, Abrahams DA, Lerumo L, et al. Functional Capacity of Mycobacterium tuberculosis-Specific T Cell Responses in Humans Is Associated with mycobacterial load. J Immunol 2011;187:2222-32. 